\title{
ON POSITIVE SOLUTIONS OF THE HEAT EQUATION
}

\author{
MASASUMI KATO1)
}

To Professor Kiyoshi Noshiro on the occasion of his 60th birthday

1. Consider the positive and twice continuously differentiable solutions $u$ of the heat equation

$$
\left(\Delta-\frac{\partial}{\partial t}\right) u=0, \quad \Delta=\sum_{i=1}^{n} \frac{\partial^{2}}{\partial x_{i}^{2}}
$$

in an open $t$-strip $\Omega=R_{n} \times(0, T)$ for some $T>0$, where $R_{n}$ is the $n$-dimensional Euclidean space.

In this note, we prove a theorem of Fatou type on $u$ and, as its application, the uniqueness theorem for the Cauchy problem of (1).

2. The following theorem corresponds to Fatou's theorem on harmonic functions.

THeOREM 1. Let $u$ be any positive solution of (1) in $\Omega$. Then $\lim _{t \rightarrow 0+} u(x, t)$ exists for almost every $x=\left(x_{1}, x_{2}, \ldots \ldots, x_{n}\right) \in R_{n}$.

Proof. We begin with the Poisson-Stieltjes integral representation of $u(x, t)$ which is valid at least near the hyperplane $t=0$. The representation is classical when $n=1$ (cf. [5]).

Let $t_{0}$ be any fixed value such that $0<t_{0}<T$ and $\varepsilon$ be any number such that $0<\varepsilon<T-t_{0}$. Then we can represent $u(x, t+\varepsilon)$ as follows:

$$
u(x, t+\varepsilon)=\int_{R_{n}} k(y-x, t) u(y, \varepsilon) d y \quad \text { in } \Omega_{\varepsilon},
$$

where $k(y, t)=(4 \pi t)^{-n} / 2 \exp \left(-|y|^{2} / 4 t\right), \quad|y|^{2}=\sum_{i=1}^{n} y_{i}^{2}$

and $\Omega_{\varepsilon}=R_{n} \times(0, T-\varepsilon)$ (e.g. [2] p. 42-48).

Received September 12, 1966.

1) The author wishes to thank Professors K. Noshiro, T. Kuroda for their constant encouragement and to express his deep appreciation to Professor S. Ito, who kindly gave many helpful suggestions to the author. 
By choosing $x=0, t=t_{0}$ in (2), we see that the Borel measures

$$
d \mu_{\varepsilon}=k\left(y, t_{0}\right) u(y, \varepsilon) d y
$$

are uniformly bounded for sufficiently small $\varepsilon>0$. Hence, by Frostman's selection theorem, there exists a sequence $\left\{\mu_{\varepsilon_{j}}\right\}_{j=1}^{\infty}$ of Borel measures converging to some Borel measure $\mu$ as $\varepsilon_{1}>\varepsilon_{2}>\ldots \ldots>\varepsilon_{j}>\ldots \ldots \rightarrow 0$. This means that $\lim \mu_{\varepsilon_{j}}(X)=\mu(X)$ for each Borel set $X$ with the boundary of $\mu$-measure zero. Hence, for any point $(x, t)$ in $\Omega_{0}=R_{n} \times\left(0, t_{0}\right)$, we have

$$
\begin{aligned}
& u\left(x, t+\varepsilon_{j}\right)=\int_{R_{n}} k(y-x, t) u\left(y, \varepsilon_{j}\right) d y \\
& \quad=\int_{R_{n}} \frac{k(y-x, t)}{k\left(y, t_{0}\right)} d \mu_{\varepsilon_{j}} \rightarrow \int_{R_{n}} \frac{k(y-x, t)}{k\left(y, t_{0}\right)} d \mu \quad \text { as } j \rightarrow \infty,
\end{aligned}
$$

where the passage to the limit is justified by noting the choice of $\mu_{\varepsilon_{j}}$ and by the obvious estimate

$$
k(y-x, t) \mid k\left(y, t_{0}\right)=O\left(e^{- \text {const. }|y|^{2}}\right) \text { as }|y| \rightarrow \infty .
$$

By setting $d \sigma=d \mu / k\left(y, t_{0}\right)$, we obtain the desired representation

$$
u(x, t)=\int_{R_{n}} k(y-x, t) d \sigma \quad \text { in } \Omega_{0},
$$

where $\sigma$ is obviously finite for the bounded Borel sets in $R_{n}$.

Now, we consider the Lebesgue decomposition of $d \sigma$ :

$$
d \sigma=\varphi(y) d y+d s,
$$

where the density $\varphi \geqq 0$ is locally summable on $R_{n}$ and $s \geqq 0$ is singular. By the strong version of Lebesgue's theorem and by the fact that the symmetric derivative $D_{\text {sym }} s(x)$ of $s$ vanishes at almost every $x \in R_{n}$, we have

$$
a^{-n} \int_{|y-x| \leqq a}\{|\varphi(y)-\varphi(x)| d y+d s(y)\} \rightarrow 0 \quad \text { as } a \rightarrow 0
$$

for almost every $x \in R_{n}$.

We may assume that (4) holds for $x=0$. Then, for any $\varepsilon>0$, there exists $a_{0}>0$ such that the left hand side of (4) is less than $\varepsilon$ whenever $0<a \leqq 2 a_{0}$. Moreover, for any $t$ such that $0<t<\operatorname{Min}\left(a_{0}^{2}, t_{0}\right)$ we choose a positive integer $N$ such that

$$
2^{N-1} b \leqq a_{0}<2^{N} b, \quad b=t^{1 / 2}
$$

By (3), we see 


$$
\begin{aligned}
& |u(0, t)-\varphi(0)| \leqq \\
& \quad\left(\int_{|y|<b}+\sum_{i=1}^{N} \int_{2^{j-1} b \leqq|y|<2^{j b}}+\int_{|y|>a_{0}}\right) k(y, t)\{|\varphi(y)-\varphi(0)| d y+d s(y)\} .
\end{aligned}
$$

Estimating each integral, we see easily that

$$
\begin{aligned}
& |u(0, t)-\varphi(0)| \leqq \text { Const. } \varepsilon+\text { Const. } \varepsilon \sum_{j=1}^{\infty} 2^{-n j} \\
& \quad+\varphi(0) \int_{|y|>a_{0}} k(y, t) d y+\mu\left(R_{n}\right) t_{0}^{n / 2} e^{a_{0}^{2} / 4 t_{0}} t^{-n / 2} e^{-a_{0}^{2} / 4 t} \\
& \quad \leqq \text { Const. } \varepsilon \text { as } t \rightarrow 0+,
\end{aligned}
$$

which proves our assertion.

Remark. P.C. Rosenbloom ([3], p. 191-200) remarked without proof the validity of Fatou's theorem under a somewhat strong growth condition about $u(x, t)$.

3. Here, with the aid of Theorem 1, we prove the uniqueness theorem for positive solutions under some weak conditions (cf. [5] and [3]). For the purpose, we prepare the following lemma.

Lemмa. If $\sigma \geqq 0$ is a Borel measure on $R_{n}$ and if the upper symmetric derivative $\bar{D}_{\operatorname{sym} \sigma(x)}$ is finite at each point $x \in R_{n}$, then $\sigma$ is absolutely continuous with respect to the n-dimensional Lebesgue measure.

Although we could deduce its proof from Ward's decomposition theorem ([4], p. 151-152), we state here a direct proof along the way suggested by Prof. S. Ito.

Proof. Assume that there exists a compact set $K$ such that $\sigma(K)>0$ and such that the Lebesgue measure $|K|$ of $K$ equals zero. Then, for a sufficiently large $M>0$, there exists a compact subset $K_{0}$ of $K$ such that $\sigma\left(K_{0}\right)>0$ and $\sigma(S) / r^{n} \leqq M$ whenever $S$ is a closed sphere with center in $K_{0}$ and of radius $r$ less than $M^{-1}$. On the other hand, on account of $\left|K_{0}\right|=0$, there exists a sequence of open cubes $\left\{I_{j}\right\}_{j=1}^{\infty}$ such that

(a) the diameter $a_{j}$ of $I_{j}$ is less than $M^{-1}$,

(b) $\bigcup_{j=1}^{\infty} I_{j} \supset K_{0}$ and

(c) $\sum_{j=1}^{\infty}\left|I_{j}\right|<\left(n^{n / 2} M\right)^{-1} \sigma\left(K_{0}\right)$.

By (b) and (c), we have, for at least one $I_{j_{0}}$,

$$
\left|I_{j_{0}}\right|<\left(n^{n / 2} M\right)^{-1} \sigma\left(K_{0} \cap I_{j_{0}}\right) .
$$


Considering a closed sphere $S_{0}$ with center in $K_{0} \cap I_{j_{0}} \neq \phi$ and of radius $a_{j_{0}}$, we have a contradiction.

Now we can prove the following.

Theorem 2. If $\lim \sup _{t \rightarrow 0+} u(x, t)<+\infty$ at each point $x \in R_{n}$ and if $\lim _{t \rightarrow 0+} u(x, t)$ $=0$ for almost every $x \in R_{n}$, then $u=0$ in $\Omega$.

Proof. Assume that, for some point, e.g., $x=0$, we have $\bar{D}_{\text {sym } \sigma}(0)=+\infty$. Then, there exists a sequence of radii $\left\{r_{j}\right\}_{j=1}^{\infty}$ converging to zero such that

$$
\lim \sigma\left(S_{j}\right) / r_{j}^{n}=+\infty,
$$

where $S_{j}$ denotes the closed sphere with center $x=0$ and of radius $r_{j}$. Thus, for a sufficiently large $j$, we have by (3)

$$
u\left(0, r_{j}^{2}\right) \geqq \int_{|y| \leqq r_{j}} k\left(y, r_{j}^{2}\right) d \sigma \geqq \text { Const. } \sigma\left(S_{j}\right) / r_{j}^{n},
$$

which, by letting $j \rightarrow+\infty$, leads us to a contradiction.

Hence, by the above lemma, $\sigma$ is absolutely continuous, that is, $s=0$. On the other hand, by our assumption and Theorem 1 , we see that $\varphi(x)=0$ for almost every $x \in R_{n}$. Thus, we have $\sigma=0$, that is, $u(x, t)=0$ in $\Omega_{0}$. Since $t_{0}$ is arbitrary in $(0, T)$, we conclude that $u(x, t)=0$ in $\Omega$.

4. By replacing $k(y, t)$ by the fundamental solution given in [1], we can replace Laplacian $\Delta$ in our theorems by an elliptic differential operator $A$ of the following form:

$$
A=\sum_{i, j} a_{i j}(x) \frac{\partial^{2}}{\partial x_{i} \partial x_{j}}+\sum_{i} b_{i}(x) \frac{\partial}{\partial x_{\imath}}+c(x),
$$

where the matrix $\left(a_{i j}(x)\right)$ is symmetric and strictly positive definite for any $x \in R_{n}$ and

$$
D^{2} a_{i j}(x), D a_{i j}(x), D b_{i}(x), c(x) \text { and } \operatorname{det}\left(a_{i j}(x)\right)^{-1}
$$

are bounded and Hölder continuous on $R_{n}{ }^{2)}$

\section{REFERENCES}

[1] F.G. Dressel: The fundamental solution of the parabolic equation. Duke Math. J., 7 (1940) 186-203, 13(1946) 61-70.

[2] A. Friedman: Partial differential equations of parabolic type. Prentice-Hall (1964).

[3] P.C. Rosenbloom: Linear equations of parabolic type with constant coefficients. Contribu-

2) For this remark the author owes to Prof. S. Ito. 
tions to the theory of partial differential equations. Ann. Math. Studies. no. 33 (Princeton Univ. Press, 1954), p. 191-200.

[4] S. Saks: Theory of the integral. Warsaw. (1937).

[5] D.V. Widder: Positive temperatures on an infinite rod. Tran. Amer. Math. Soc., 55, 85-95 (1944).

Suzuka College of Technology 